Chinese Journal of Organic Chemistry

REVIEW

\title{
硝基唑类含能化合物的合成研究进展
}

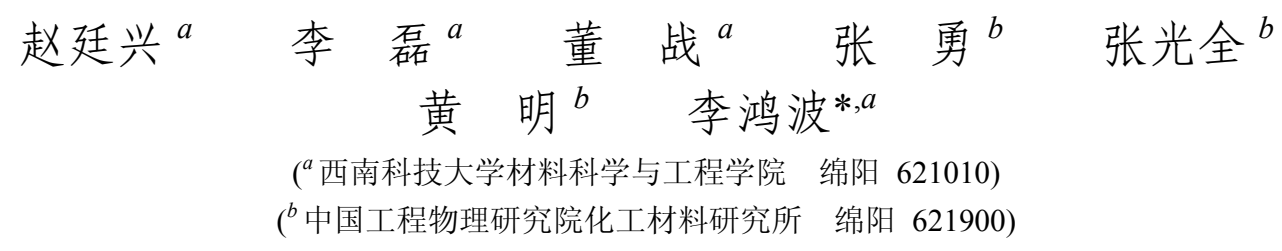

\begin{abstract}
摘要 硝基唑类化合物在含能材料领域中应用较为广泛. 按直接硝化、间接硝化、硝基重排、其他方法将硝化方法进 行分类, 综述了吡唑、咪唑、三唑及四唑硝基衍生物的合成, 并介绍了一些重要硝基唑类含能化合物的特性及主要应 用.

关键词 硝基; 唑; 含能化合物; 合成; 进展
\end{abstract}

\section{Research Progress on the Synthesis of Energetic Nitroazoles}

\author{
Zhao, Tingxing ${ }^{a}$ \\ $\mathrm{Li}, \mathrm{Lei}^{a}$ \\ Dong, Zhan ${ }^{a}$ \\ Zhang, Yong ${ }^{b}$ \\ Zhang, Guangquan ${ }^{b}$ \\ Huang, Ming ${ }^{b}$ Li, Hongbo*,a
}

( ${ }^{a}$ School of Material Science and Engineering, Southwest University of Science and Technology, Mianyang 621010)

( ${ }^{b}$ Institute of Chemical Materials, China Academy of Engineering Physics, Mianyang 621900)

\begin{abstract}
Nitroazoles are widely used in energetic materials. Classified into direct nitration, indirect nitration, nitro rearrangement and other methods, the nitration methods for achieving nitro-derivatives of pyrazole, imidazole, triazole and tetrazole are summarized. The characteristics and main applications of some important energetic nitroazoles are also introduced.
\end{abstract}

Keywords nitryl; azole; energetic compounds; synthesis; progress

随着国防、科技及工业发展的需要, 高能低(钝)感 化合物越来越受到含能材料工作者的重视 ${ }^{[1]}$. 大量研究 工作表明, 硝基唑类化合物分子结构中含有 $\mathrm{N}-\mathrm{N}, \mathrm{C}-$ $\mathrm{N}, \mathrm{N}-\mathrm{O}$ 等高能化学键, 具有高氮低碳氢的特点, 使此 类化合物具有较高的密度, 且更易达到氧平衡, 是一类 新型的高能化合物, 其中部分化合物具备了优良含能材 料的高能低(钝)感特性 ${ }^{[2]}$. 由于唑类氮杂环分子结构的 紧密性、稳定性及可改良性等优点, 使得以其为母体的 硝化、衍生等工作相对较为容易, 诸多硝基唑类含能化 合物相继被合成出来 ${ }^{[3]}$, 在高能钝感炸药、推进剂、烟 火剂及其他领域有了较好的应用.

本文按硝化方法进行分类, 综述了吡唑、咪唑、三 唑及四唑硝基衍生物的合成. 其中包括: 直接硝化法、 间接硝化法、硝基重排法及其他方法, 并对其中一些较
为重要唑类含能化合物的特性和主要应用进行了介绍.

\section{1 直接硝化法}

直接硝化法常以不同浓度的 $\mathrm{HNO}_{3}$, 不同体积比的 $\mathrm{HNO}_{3} / \mathrm{H}_{2} \mathrm{SO}_{4}$ 或 $\mathrm{HNO}_{3}$ /酸䣶混合液, $\mathrm{NO}_{2} \mathrm{BF}_{4}, \mathrm{NO}_{2} / \mathrm{O}_{3}$ 或 $\mathrm{N}_{2} \mathrm{O}_{5}$, 硝酸盐等作为硝化试剂. 这些硝化试剂可自身或 间接生成硝酰正离子 $\left(\mathrm{NO}_{2}^{+}\right)$, 具有硝化能力的 $\mathrm{NO}_{2}^{+}$与 唑环可发生芳香亲电取代反应，从而制得相应的硝基唑 类衍生物. 其机理见 Scheme 1.

\section{$1.1 \mathrm{HNO}_{3}$ 为硝化试剂}

$\mathrm{HNO}_{3}$ 价廉宜得, 是一种应用较为广泛的硝化试剂, 对不同唑环进行硝化，需用不同浓度的 $\mathrm{HNO}_{3}$. 如由 $70 \% \mathrm{HNO}_{3}$ 直接硝化 1,2,4-三唑酮-5 (1, TO) 可制得 3-硝

\footnotetext{
*E-mail: li-honggg@163.com

Received July 30, 2013; revised September 27, 2013; published online October 28, 2013.

Project supported by the National Key Projects (No. 00402040103) and the Youth Innovation Research Team of Sichuan for Carbon Nanomaterials (No. 2011JTD0017).

国家重大科技专项(No. 00402040103)和碳纳米材料四川省青年科技创新研究团队专项(No. 2011JTD0017)资助项目.
} 


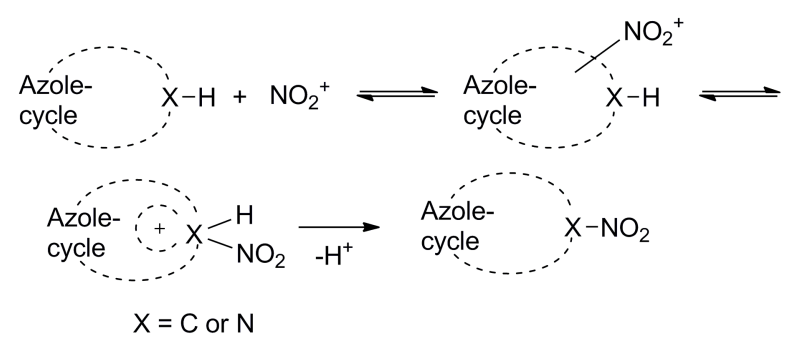

Scheme 1

基-1,2,4-三唑酮-5 (2, NTO) (Eq. 1) ${ }^{[4]}$. NTO 是备受关注 的高能钝感材料, 其生成焓为 $101.1 \mathrm{~kJ} / \mathrm{mol}$, 密度为 1.93 $\mathrm{g} / \mathrm{cm}^{3}$, 爆轰性能与 $1,3,5,7$-四硝基-1,3,5,7-四氮杂环辛烷 (奥克托今, HMX)相当且优于环三亚甲基三硝胺(黑索 今, RDX), 撞感特性落高 $H_{50}>280 \mathrm{~cm}$ (2.5 kg 落锤), 但 $\mathrm{NTO}$ 的酸性 $\left(\mathrm{p} K_{\mathrm{a}}=3.67\right)$ 限制了其应用, 其盐可作为含能 燃烧催化剂及高性能炸药 ${ }^{[4,5]}$.<smiles></smiles>

由 $100 \% \mathrm{HNO}_{3}$ 直接硝化 3,3'-偶氮-1,2,4-三唑(3)制 得 1,1'-二硝基-3,3'-偶氮-1,2,4-三唑(4, N-DNTA) (Eq. $2)^{[6]}$. N-DNTA 具有较高的能量, 较低的静电感度、冲击 感度及摩擦感度. 主要用于低特征信号推进剂、气体发 生剂、低烟或无烟烟火剂及高能炸药 ${ }^{[6]}$.<smiles>O=[N+]([O-])c1nc(N=Nc2ncn([N+](=O)[O-])n2)n(-n2cnc(N=Nc3nc[nH]n3)n2)n1</smiles>

\section{$1.2 \mathrm{HNO}_{3} / \mathrm{H}_{2} \mathrm{SO}_{4}$ 为硝化试剂}

$\mathrm{HNO}_{3} / \mathrm{H}_{2} \mathrm{SO}_{4}$ 的硝化能力较 $\mathrm{HNO}_{3}$ 强, 常用于多种 唑类化合物的硝化. 根据不同唑环的结构特点和发生芳 香亲电取代反应难易程度, 可选择合适配比的 $\mathrm{HNO}_{3} / \mathrm{H}_{2} \mathrm{SO}_{4}$ 进行硝化, 反应条件大多比较温和, 所需 温度一般为 $20 \sim 100{ }^{\circ} \mathrm{C}$, 反应时间约 $1 \sim 2.5 \mathrm{~h}$, 产率可 达 $80 \%$ 左右或更高, 对于一些反应活性较为惰性的唑环 底物(如强吸电子基存在), 反应速率一般较慢, 反应时 间需延长至 $10 \sim 48 \mathrm{~h}$.

4-硝基吡唑(6)作为一种吡唑类硝基衍生物, 虽不能 作为炸药, 但已具备一定能量, 是合成多硝基吡唑类化 合物的重要中间体, 其可由体积比为 $1: 3$ 的 $\mathrm{HNO}_{3} / \mathrm{H}_{2} \mathrm{SO}_{4}$ 硝化吡唑 5 制得(Eq. 3) ${ }^{[7]}$.

3-硝基吡唑 7 经体积比约为 $1: 3$ 的 $\mathrm{HNO}_{3} / \mathrm{H}_{2} \mathrm{SO}_{4}$ 硝化可制得 3,4-二硝基吡唑(8, 3,4-DNP) (Eq. 4) ${ }^{[7 \mathrm{a}]}$. 3,4-DNP 的熔点 $91 \sim 92{ }^{\circ} \mathrm{C}$ 、氮含量 $35.44 \%$ 、氧平衡

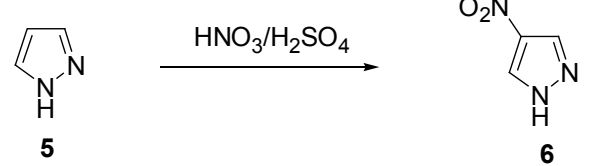

$-30.33 \%$ 、密度 $1.81 \mathrm{~g} / \mathrm{cm}^{3}$ 、爆速 $8.10 \mathrm{~km} / \mathrm{s}$ 、爆压 $2.94 \times 10^{7} \mathrm{~Pa} 、 H_{50}=146.9 \mathrm{~cm}$ ( $2.5 \mathrm{~kg}$ 落锤), 能作为优良 熔铸炸药的载体, 用于混合炸药. 3,4-DNP 与 RDX 以质 量比 $40: 60$ 混合, 爆速为 $8.69 \mathrm{~km} / \mathrm{s}$, 与 HMX 以质量比 $30: 70$ 混合, 爆速可达 $9.01 \mathrm{~km} / \mathrm{s}^{[8]}$.<smiles>O=[N+]([O-])c1c[nH]nc1[N+](=O)[O-]</smiles>

采用体积比 $1: 2$ 的 $\mathrm{HNO}_{3} / \mathrm{H}_{2} \mathrm{SO}_{4}$ 直接硝化 3,5-二硝 基吡唑(9)制得 3,4,5-三硝基吡唑(10, 3,4,5-TNP)(Eq. 5) ${ }^{[9]}$, 其密度为 $2.00 \mathrm{~g} / \mathrm{cm}^{3}$ 、爆速 $9.40 \mathrm{~km} / \mathrm{s}$ 、爆压 $4.16 \times 10^{7}$ $\mathrm{Pa}^{[10]}$. 以体积比 $1: 4$ 的 $\mathrm{HNO}_{3} / \mathrm{H}_{2} \mathrm{SO}_{4}$ 于 $110{ }^{\circ} \mathrm{C}$ 直接硝 化 1-甲基吡唑(11)可顺利制得 1-甲基-3,4,5-三硝基吡唑 $\left(12\right.$, MTNP)(Eq. 6), 其熔点为 $91{ }^{\circ} \mathrm{C}$ 、分解温度 $256{ }^{\circ} \mathrm{C}$ 、 密度 $1.83 \mathrm{~g} / \mathrm{cm}^{3}$ 、爆速 $8.96 \mathrm{~km} / \mathrm{s}$ 、爆压 $3.35 \times 10^{7} \mathrm{~Pa}^{[11]}$. 10 和 12 均可替代 2,4,6-三硝基甲苯(TNT)用于熔铸炸药, 也可用于高能低感炸药及推进剂配方 ${ }^{[2 a]}$.<smiles>O=[N+]([O-])c1cc([N+](=O)[O-])[nH]n1</smiles><smiles>O=[N+]([O-])c1n[nH]c([N+](=O)[O-])c1[N+](=O)[O-]</smiles>

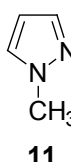<smiles>Cn1nc([N+](=O)[O-])c([N+](=O)[O-])c1[N+](=O)[O-]</smiles>

12 (MTNP)

4,5-二硝基咪唑 $(15,4,5-\mathrm{DNI})$ 是一种高能针感炸药 $\left(H_{50}>200 \mathrm{~cm}, 2.5 \mathrm{~kg}\right.$ 落锤), 由于环上两个硝基的强吸 电特性，导致 NH 显酸性，其应用受到一定限制. 15 与 氨反应所制得的铵盐, 可作为燃烧催化剂, 用于推进剂 中 ${ }^{[12]}$. 咪唑 13 经含 $70 \% \mathrm{HNO}_{3}$ 的 $\mathrm{HNO}_{3} / \mathrm{H}_{2} \mathrm{SO}_{4}$ 硝化制得 4-硝基咪唑(14) ${ }^{[13]}$, 以体积比为 $4: 5$ 的 $\mathrm{HNO}_{3} / \mathrm{H}_{2} \mathrm{SO}_{4}$ 对 其进行深度硝化制得 15 (Scheme 2) ${ }^{[14]}$.

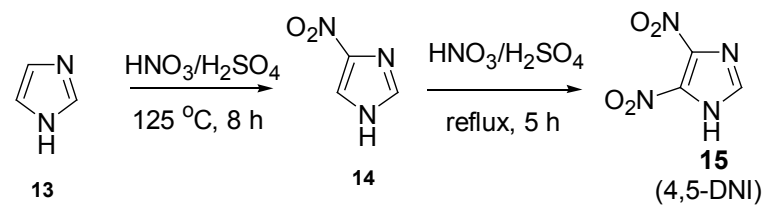

Scheme 2 
2-硝基咪唑(16)或 2,4-二硝基咪唑(17, 2,4-DNI)经不 同配比的 $\mathrm{HNO}_{3} / \mathrm{H}_{2} \mathrm{SO}_{4}$ 硝化均可制得 2,4,5-三硝基咪唑 $(\mathbf{1 8}, 2,4,5-\mathrm{TNI})$ (Scheme 3) ${ }^{[13]}$, 其熔点为 $248{ }^{\circ} \mathrm{C}$ 、标准生 成焓 $229.5 \mathrm{~kJ} / \mathrm{mol}$ 、密度 $1.93 \mathrm{~g} / \mathrm{cm}^{3}$ 、爆速 $8.73 \mathrm{~km} / \mathrm{s}$ 、爆 压 $3.21 \times 10^{7} \mathrm{~Pa}$, 感度低于 RDX 与 $\mathrm{HMX}$, 其铵盐能量与 $\mathrm{RDX}$ 相近, 但更加稳定, 可与其他炸药混合成低共融混 合物, 有效提高炸药的综合性能 ${ }^{[15]}$.

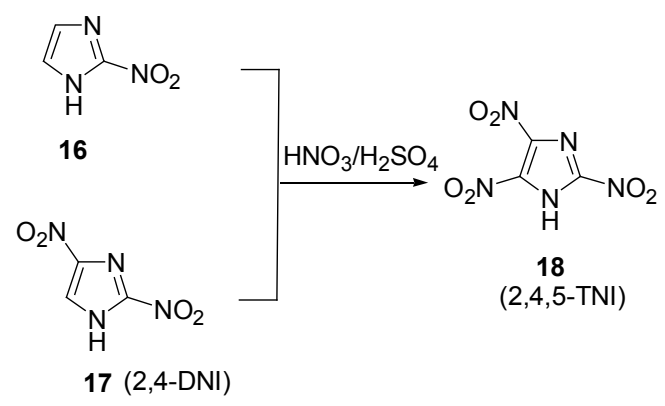

Scheme 3

2-(2,4,6-三硝基苯基)-4-硝基-1,2,3-三唑(24)作为一 种三唑类硝基衍生物, 其熔点为 $166{ }^{\circ} \mathrm{C}$, 密度 1.75 $\mathrm{g} / \mathrm{cm}^{3} .24$ 可由 2-苯基-1,2,3-三唑(19)为原料经连续硝化 制得, 也能以体积比 $1: 2$ 的 $\mathrm{HNO}_{3} / \mathrm{H}_{2} \mathrm{SO}_{4}$ 硝化 2-(2,4,6三硝基苯基)-1,2,3-三唑(23)制得(Scheme 4) ${ }^{[16]}$.

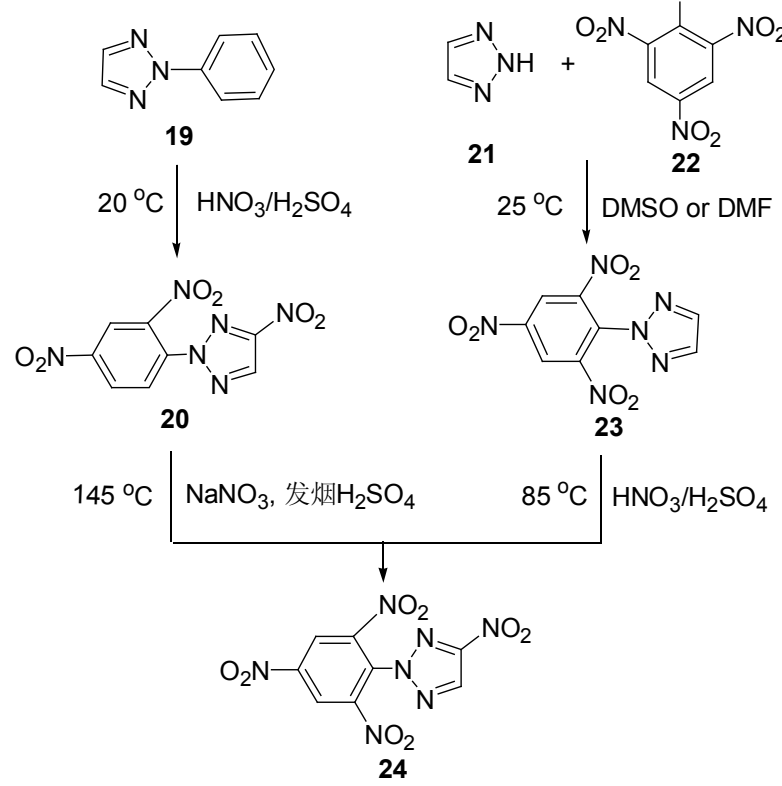

Scheme 4

\section{$1.3 \mathrm{HNO}_{3}$ /酸酐为硝化试剂}

与硝化试剂 $\mathrm{HNO}_{3} / \mathrm{H}_{2} \mathrm{SO}_{4}$ 相比, $\mathrm{HNO}_{3}$ /酸䣫混合液 的硝化能力较弱, 但酸跴能有效降低 $\mathrm{HNO}_{3}$ 的氧化性, 在使用过程中仍具其独特性. 如 3,5-二甲基吡唑(25)经 硝酸与三氟乙酸酩 $\left(\mathrm{HNO}_{3} / \mathrm{TFAA}\right)$ 选择性硝化制得 $3,5-$ 二
甲基-4-硝基吡唑(26) (Eq. 7) ${ }^{[17]}$.<smiles>Cc1cc(C)[nH]n1</smiles>

$\mathrm{HNO}_{3} /$ TFAA 硝化 $\mathbf{5}$ 制得 8 (3,4-DNP) ${ }^{[17]}, \mathbf{8}$ 由 $\mathrm{NH}_{4} \mathrm{NO}_{3}$ /TFAA 进一步硝化可制得 1,3,4-三硝基吡唑(27, 1,3,4-TNP) (Scheme 5) ${ }^{[18]}$.

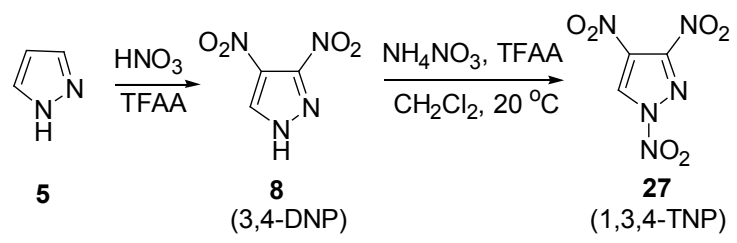

Scheme 5

咪唑类硝基衍生物，如 1,4-二硝基咪唑 (28, 1,4-DNI)可由 13 或 14 经 $\mathrm{HNO}_{3} / \mathrm{Ac}_{2} \mathrm{O}$ 硝化制得(Scheme $6)^{[19,20]}$.

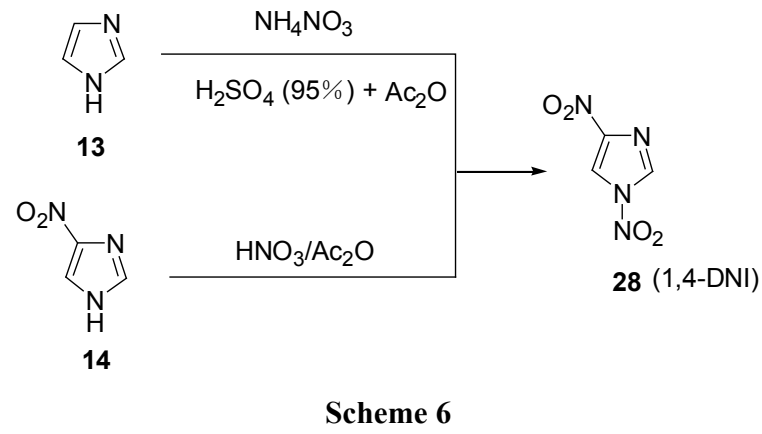

16 经 $\mathrm{HNO}_{3} / \mathrm{Ac}_{2} \mathrm{O}$ 硝化制得 17 (2,4-DNI) (Eq. 8) ${ }^{[20]}$, 其密度为 $1.77 \mathrm{~g} / \mathrm{cm}^{3}$ 、爆速 $8.13 \mathrm{~km} / \mathrm{s}$ 、爆压 $2.81 \times 10^{7} \mathrm{~Pa}$ 、 感度低于 RDX 和 HMX, 具有比 1,3,5-三氨基-2,4,6-三硝 基苯(TATB)高 15\% 20\%的能量 ${ }^{[19 a]}$. 2,4-DNI 是高能针 感含能材料候选物之一, 也是合成 $\mathbf{1 8}(2,4,5-\mathrm{TNI}) 、 1$-甲 基-2,4,5-三硝基咪唑(MTNI)等含能材料的一种重要中 间体 ${ }^{[16,13]}$.

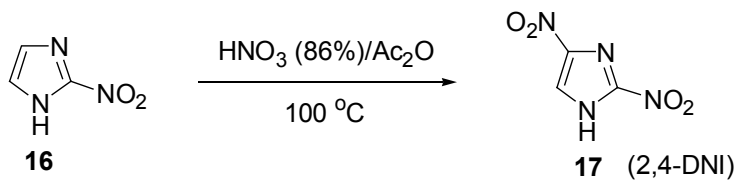

3-氨基-1,2,4-三唑(29)经 $\mathrm{HNO}_{3} / \mathrm{Ac}_{2} \mathrm{O}$ 在不同的条件 下选择性硝化可制备 1-硝基-3-氨基-1,2,4-三唑(30)和 3氨基-5-硝基-1,2,4-三唑(31，ANTA $)^{[21]}$. ANTA 熔点 238 ${ }^{\circ} \mathrm{C}$ 、密度 $1.82 \mathrm{~g} / \mathrm{cm}^{3}$ 、爆速 $8.46 \mathrm{~km} / \mathrm{s} 、 H_{50}>320 \mathrm{~cm}(2.5$ $\mathrm{kg}$ 落锤), 与 TATB 相当, 可用作钝感炸药的候选组 
分 ${ }^{[22]}$; 也可作为制备一些重要炸药的中间体, 如 4,6-双 (5-氨基-3-硝基-1H-1,2,4-三唑-1-基)-5-硝基嘧啶 (33, DANTNP)，3,6-双 (5- 氨基-3-硝基-1H-1,2,4-三唑-1基)-1,2,4,5-四嗪(34)等耐热炸药(Scheme 7) ${ }^{[22]}$.

此外, 4 ( $N$-DNTA) 也可通过 $\mathrm{HNO}_{3}$ /酸酐法制得(Eq. 9) ${ }^{[23]}$.

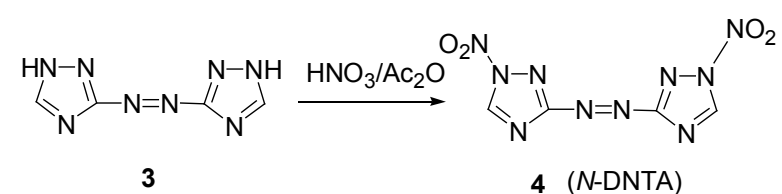

\section{$1.4 \mathrm{NO}_{2} \mathrm{BF}_{4}$ 为硝化试剂}

$\mathrm{NO}_{2} \mathrm{BF}_{4}$ 是一种对环境友好的硝化试剂, 无需废酸 处理; 反应条件比较温和, 温度易控制; 无氧化性, 副 产物较少, 反应选择性较高; 其可用于各种唑环的芳香 亲电硝化反应, 反应温度 $0 \sim 20{ }^{\circ} \mathrm{C}$, 常以乙腈为溶剂, 反应时间一般为 $10 \mathrm{~h}$, 产率 30\% 50\%. 如 8 (3,4-DNP) 在乙腈溶剂中经 $\mathrm{NO}_{2} \mathrm{BF}_{4}$ 硝化可制得 27 (1,3,4-TNP) (Eq. 10) ${ }^{[24]}$.<smiles>O=[N+]([O-])c1c[nH]nc1[N+](=O)[O-]</smiles>

以硝基甲烷为溶剂, 1 -甲基咪唑(35)与 $\mathrm{NO}_{2} \mathrm{BF}_{4}$ 经 $400 \mathrm{~W}$ 微波照射 $3 \mathrm{~min}$ 即可制得 1-甲基-2,4,5-三硝基咪 唑(36, MTNI)(Eq. 11) ${ }^{[25]}$. 13, 2-氯咪唑(37)或 1,2,4-三唑 (38)，5-氯-1,2,4-三唑(39)经 $\mathrm{NO}_{2} \mathrm{BF}_{4}$ 硝化分别制得 1-硝 基咪唑(40), 1-硝基-2-氯咪唑(41), 1-硝基-1,2,4-三唑(42), 5-氯-1-硝基-1,2,4-三唑(43) (Eq. 12) ${ }^{[26]}$.

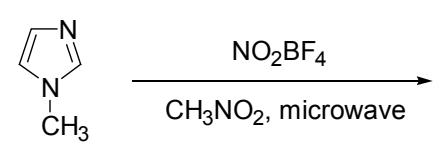

35<smiles></smiles><smiles>[R]c1cn[nH]c1CC[N+]([O-])(O)C(C)C</smiles>

$13 \mathrm{X}=\mathrm{CH} ; \mathrm{R}=\mathrm{H}$

$37 \mathrm{X}=\mathrm{CH} ; \mathrm{R}=\mathrm{Cl} \quad 40 \mathrm{X}=\mathrm{CH} ; \mathrm{R}=\mathrm{H}$

$38 \mathrm{X}=\mathrm{N} ; \mathrm{R}=\mathrm{H}$

$39 \mathrm{X}=\mathrm{N} ; \mathrm{R}=\mathrm{Cl}$

$41 \mathrm{X}=\mathrm{CH} ; \mathrm{R}=\mathrm{Cl}$

$42 \mathrm{X}=\mathrm{N} ; \mathrm{R}=\mathrm{H}$

$43 \mathrm{X}=\mathrm{N} ; \mathrm{R}=\mathrm{Cl}$

31 (ANTA) 也可由 $\mathrm{NO}_{2} \mathrm{BF}_{4}$ 硝化 29 制得，与前述的 $\mathrm{HNO}_{3}$ /酸酐法相比, 该反应条件更温和, 无需废酸处理 (Eq. 13) ${ }^{[27]}$. 而 5-氨基-1,2,4-三唑(44)经 $\mathrm{NO}_{2} \mathrm{BF}_{4}$ 硝化制 得 1-硝基-5-氨基-1,2,4-三唑(45) (Eq. 14) ${ }^{[21]}$.
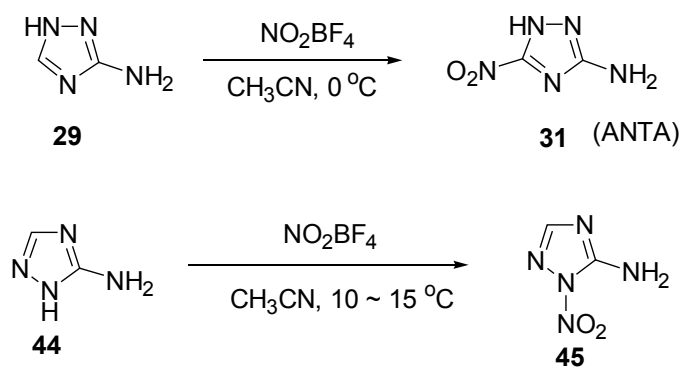

\section{$1.5 \mathrm{NO}_{2} / \mathrm{O}_{3}$ 为硝化试剂}

$\mathrm{NO}_{2}$ 本身几乎无硝化能力, 需 $\mathrm{O}_{3}$ 活化后才具有硝 化能力. 此硝化方法具有环境友好、反应条件易控制、 硝化选择性高等优点, 常用于吡唑或咪唑化合物中 1-位 和 4-位的定向硝化 ${ }^{[28]}$, 反应温度一般为 $0{ }^{\circ} \mathrm{C}$, 时间约 $1 \sim 4 \mathrm{~h}$, 产率 $80 \%$ 左右或更高. 如 5 经 $\mathrm{NO}_{2} / \mathrm{O}_{3}$ 硝化可制

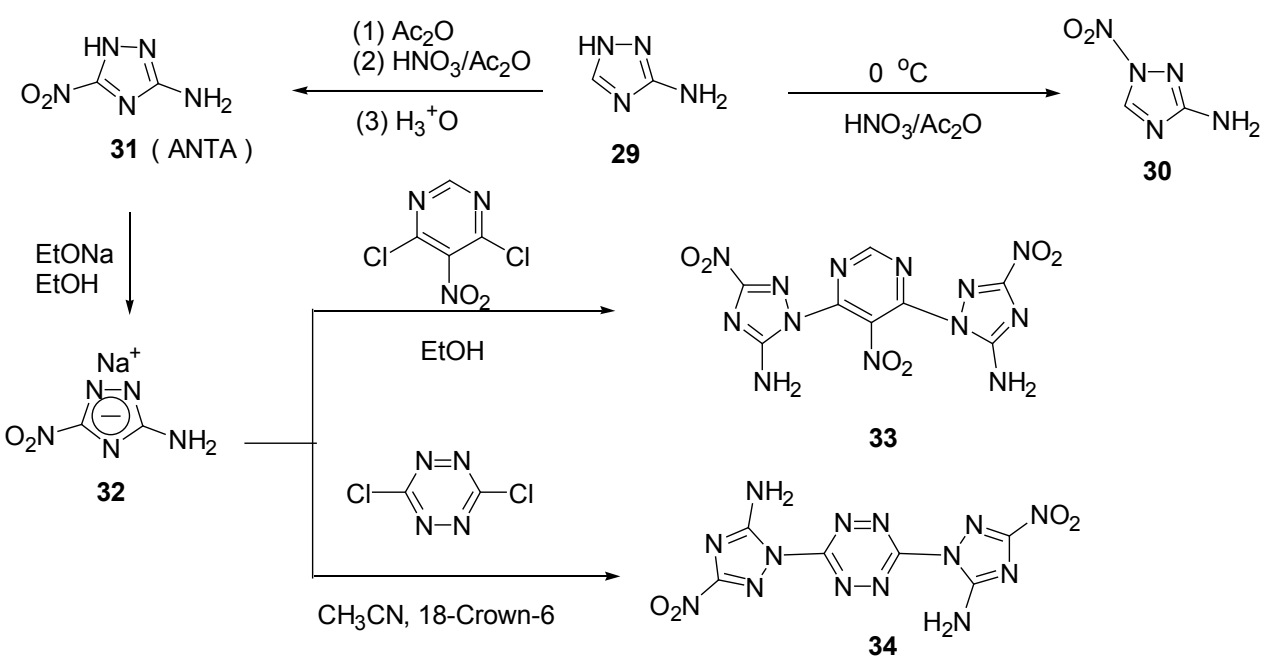

Scheme 7 
得 1-硝基吡唑(46), 再经 $\mathrm{NO}_{2} / \mathrm{O}_{3}$ 二次硝化又可制得 1,4二硝基吡唑(47, 1,4-DNP) ${ }^{[29]}$. 此外, 6 经 $\mathrm{NO}_{2} / \mathrm{O}_{3}$ 硝化也 可制得 47 (Scheme 8) ${ }^{[29]}$.

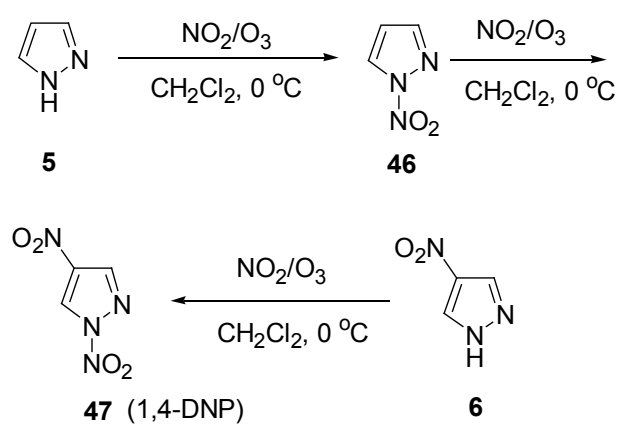

\section{Scheme 8}

采用 $\mathrm{NO}_{2} / \mathrm{O}_{3}$ 对咪唑 13 和各种甲基咪唑 $48 \sim 50$ 进 行硝化, 往往在分子中 1-位或 4-位选择性的引入硝基制 得 14、40 和 $51 \sim 56$ (Eq. 15) ${ }^{[29]}$.

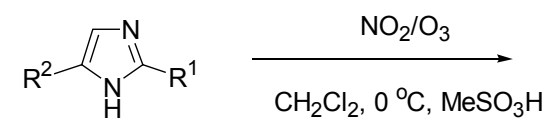

$13 \mathrm{R}^{1}, \mathrm{R}^{2}=\mathrm{H}$

$48 \mathrm{R}^{1}=\mathrm{CH}_{3}, \mathrm{R}^{2}=\mathrm{H}$

$49 \mathrm{R}^{1}=\mathrm{H}, \mathrm{R}^{2}=\mathrm{CH}_{3}$

$50 \mathrm{R}^{1}=\mathrm{CH}_{3}, \mathrm{R}^{2}=\mathrm{CH}_{3}$<smiles>[R]c1nc([N+](=O)[O-])c([R])[nH]1</smiles>

$$
\begin{array}{ll}
14 R^{1}, R^{2}=H & 40 R^{1}, R^{2}=H \\
51 R^{1}=C_{3}, R^{2}=H & 54 R^{1}=C_{3}, R^{2}=H \\
52 R^{1}=H, R^{2}=C H_{3} & 55 R^{1}=H, R^{2}=C_{3} \\
53 R^{1}=C_{3}, R^{2}=C_{3} & 56 R^{1}=C_{3}, R^{2}=C_{3}
\end{array}
$$

\section{$1.6 \mathrm{~N}_{2} \mathrm{O}_{5}$ 为硝化试剂}

$\mathrm{N}_{2} \mathrm{O}_{5}$ 是一种绿色硝化试剂, 可用于敏感化合物的 硝化, 与 $\mathrm{HNO}_{3}, \mathrm{HNO}_{3} / \mathrm{H}_{2} \mathrm{SO}_{4}, \mathrm{HNO}_{3}$ 酸酐等硝化试剂相 比具有以下优点: 无需废酸处理; 反应放热少, 温度易 控制; 后处理简单，只需蒸出溶剂; 副反应少，产率 高 ${ }^{[28]}$. 其反应常以 $\mathrm{CH}_{2} \mathrm{Cl}_{2}$ 为溶剂, 温度一般为 $0 \sim 20$ ${ }^{\circ} \mathrm{C}$, 产率在 $90 \%$ 左右. 如 5 经 $\mathrm{N}_{2} \mathrm{O}_{5}$ 硝化、氯苯 $(\mathrm{PhCl})$ / 回流处理可制得 5-硝基吡唑 57 和 9 (Scheme 9) ${ }^{[30]}$. 化合 物 9 热分解点为 $316.8{ }^{\circ} \mathrm{C}$ 、密度 $1.80 \mathrm{~g} / \mathrm{cm}^{3}$ 、爆速 8.15 $\mathrm{km} / \mathrm{s}^{[31]}$, 其分子结构呈对称分布, 较稳定, 可作为单质 炸药, 9 通过异常亲核取代氢(VNS) 反应制得钝感炸药 4氨基-3,5-二硝基吡唑(59, LLM-116) (Scheme 9) ${ }^{[32]}$. 以 $1 H, 4 H-3,6$-二硝基吡唑[4,3-c]并吡唑(60, DNPP)为原料, 在 $\mathrm{CH}_{2} \mathrm{Cl}_{2}$ 中经 $\mathrm{N}_{2} \mathrm{O}_{5}$ 硝化制得 1,3,4,6-四硝基吡唑 [4,3-c] 并吡唑 $(61, \mathrm{TNPP})(\mathrm{Eq} .16)$, 其密度为 $2.20 \mathrm{~g} / \mathrm{cm}^{3}$ 、爆速
$9.25 \mathrm{~km} / \mathrm{s}$ 、爆压 $3.74 \times 10^{7} \mathrm{~Pa} 、 H_{50}=24 \mathrm{~cm}(2.5 \mathrm{~kg}$ 落 锤 $)^{[33]}$.
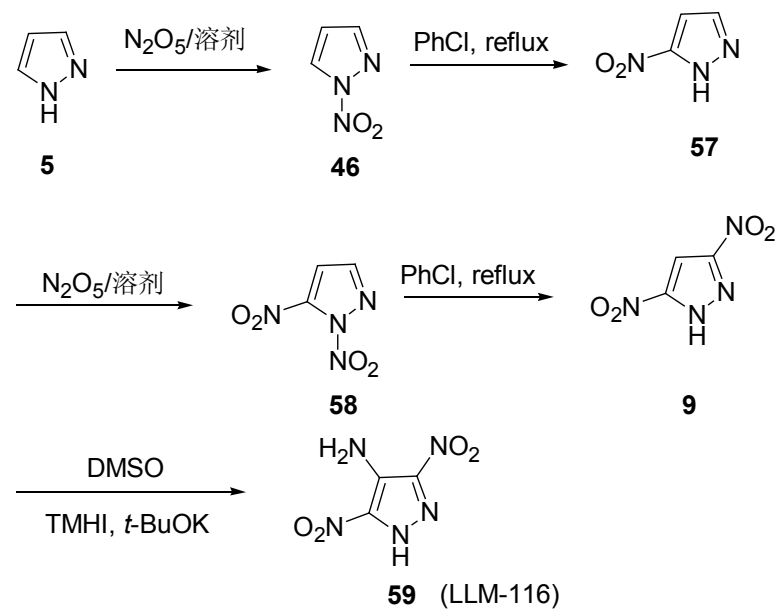

溶剂 $=\mathrm{CH}_{2} \mathrm{Cl}_{2}, \mathrm{CCl}_{4}, \mathrm{CH}_{3} \mathrm{NO}_{2}, 100 \% \mathrm{HNO}_{3}$

$\mathrm{TMHI}=1,1,1-$ 三甲基碘化肼

Scheme 9

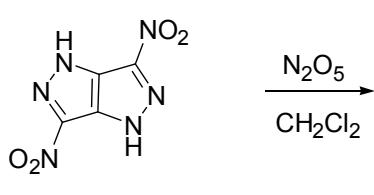

60 (DNPP)<smiles>O=[N+]([O-])c1nn([N+](=O)[O-])c2c([N+](=O)[O-])nn([N+](=O)[O-])c12</smiles>

61 (TNPP)
在 $\mathrm{CH}_{2} \mathrm{Cl}_{2}$ 溶剂中, 14 和 1-硝基咪唑(62)经 $\mathrm{N}_{2} \mathrm{O}_{5}$ 硝 化均可制得 $(\mathbf{2 8}, 1,4-\mathrm{DNI})$ (Scheme 10) ${ }^{[34,30 \mathrm{~b}]}$.

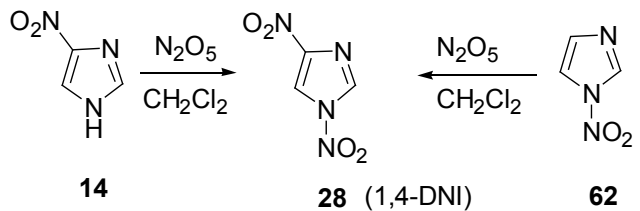

Scheme 10

2 (NTO)也可由 $\mathrm{N}_{2} \mathrm{O}_{5}$ 硝化 1 制得(Eq. 17) ${ }^{[35]}$, 与 $\mathrm{HNO}_{3}$ 硝化相比, 该反应后处理更加简单, 且无需处理 废酸.

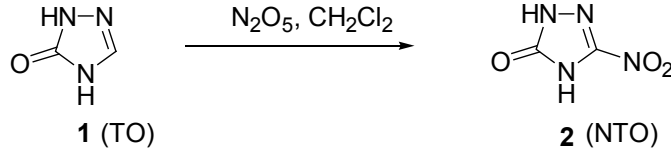

\section{7 硝酸盐为硝化试剂}

催化作用下，一些硝酸盐也可以充当硝化试剂，主 要用于一些硝基吡唑类化合物的合成，其硝化能力大小 为: $\mathrm{Bi}\left(\mathrm{NO}_{3}\right)_{3}>\mathrm{AgNO}_{3}>\mathrm{KNO}_{3}>\mathrm{NaNO}_{3}>\mathrm{NH}_{4} \mathrm{NO}_{3}>$ $\mathrm{Pb}\left(\mathrm{NO}_{3}\right)_{2}>\mathrm{Ba}\left(\mathrm{NO}_{3}\right)_{2}$. 如吡唑硝基衍生物 7, 63 和 65 的 制备(Eqs. 18 19) $)^{[36]}$. 


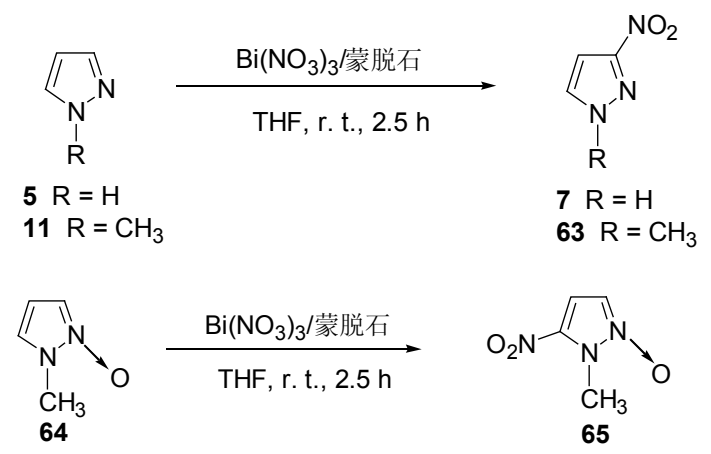

(18)

\section{2 间接硝化法}

\section{$2.1 \mathrm{NH}_{2}$ 重氮化法}

许多唑类化合物带有氨基侧链，可利用氨基的重氮 化反应引入硝基, 从而制得相应硝基唑类衍生物, 其机 理见 Scheme 11. 如 3-氨基-4-硝基吡唑(66)经重氮化反 应制得 $(\mathbf{8}, 3,4-\mathrm{DNP})\left(\right.$ Scheme 12) ${ }^{[37]}$.

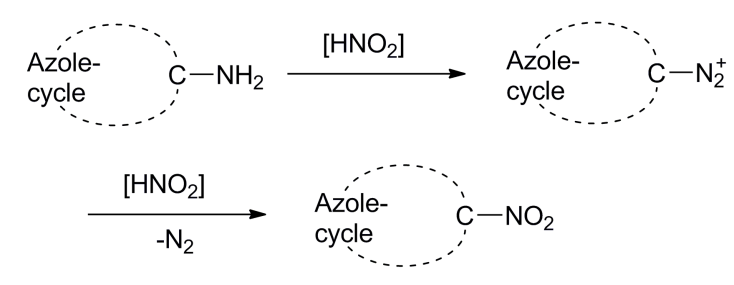

Scheme 11

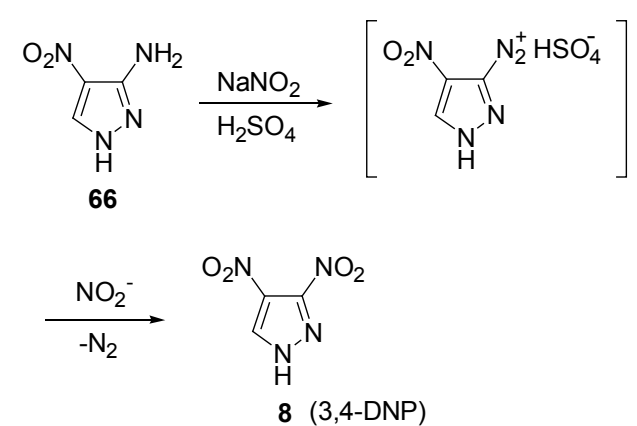

Scheme 12

1-硝脒基-3,5-二氨基-1,2,4-三唑(67)的分子结构呈 平面型, 由于空间效应及电子效应，3-位氨基较 5-位氨 基更易于发生重氮化反应，既能制备出 1-硝脒基-3-硝 基- 5-氨基-1,2,4-三唑(68, ANTA-NQ), 其密度为 1.79 $\mathrm{g} / \mathrm{cm}^{3}$ 、爆速 $8.30 \mathrm{~km} / \mathrm{s}$ 、爆压 $2.90 \times 10^{7} \mathrm{~Pa}$; 又能引入重 要的含能基团叠氮基制得化合物 69 , 其密度为 1.73 $\mathrm{g} / \mathrm{cm}^{3}$ 、爆速 $8.20 \mathrm{~km} / \mathrm{s}$ 、爆压 $2.57 \times 10^{7} \mathrm{~Pa}$ (Scheme 13) ${ }^{[34]}$.

3,5-二氨基-1,2,4-三唑(70, DAT)经重氮化反应制得 3,5-二硝基-1,2,4-三唑(71, DNT) $)^{[38]}$. DNT 与氨反应制得 3,5-二硝基-1,2,4-三唑的铵盐(72, ADNT) (Scheme $14)^{[39]}, \mathrm{ADNT}$ 热分解点为 $170{ }^{\circ} \mathrm{C}$, 热爆炸临界温度 222 ${ }^{\circ} \mathrm{C}$, 密度 $1.63 \mathrm{~g} / \mathrm{cm}^{3}{ }^{[40]}$, 是一种典型的三唑类含能化合 物, 可作为推进剂的含能消焰剂, 也是合成其他含能材

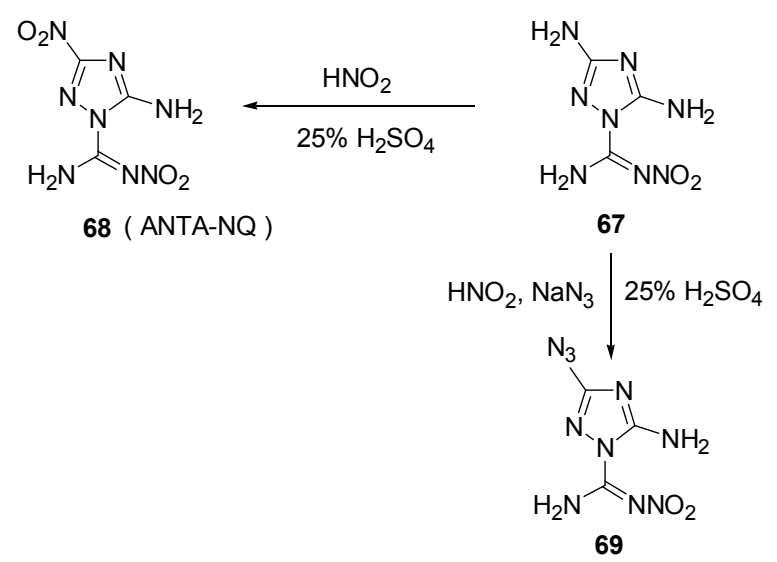

Scheme 13
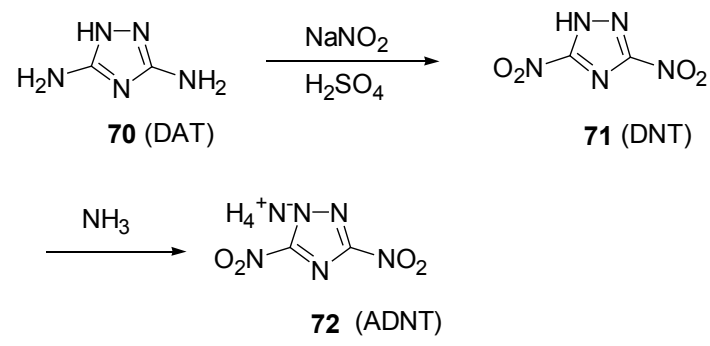

Scheme 14

料的关键中间体，如用于合成耐热炸药 33 (DANTNP)、 燃速调节剂 3,5-二硝基-1,2,4-三唑铅盐 ${ }^{[39]}$.

1-甲基-3,5-二氨基-1,2,4-三唑(73, MDAT)经重氮化 反应制得 1-甲基-3,5-二硝基-1,2,4-三唑(74, MDNT) (Eq. $20)$, 其熔点为 $98{ }^{\circ} \mathrm{C}$, 爆炸性能与 $\mathrm{RDX}$ 相当, 感度在 RDX 与 TNT 之间, 是一种优良的熔铸炸药 ${ }^{[41]}$.

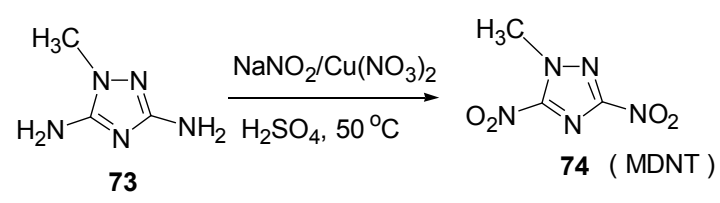

5-氨基四唑(75)经重氮化反应制得 5-硝基四唑(76) (Eq. 21) ${ }^{[42]}, 76$ 具有一定的酸性, 大多以盐或配合物形式 存在, 且其热稳定性较差, $98{ }^{\circ} \mathrm{C}$ 开始熔解, $130{ }^{\circ} \mathrm{C}$ 剧烈 分解，撞击感度与叠氮化铅相当，摩擦感度介于叠氮化 铅与四氮烯之间，爆速、爆压均高于 RDX, HMX, 与六 硝基六氮杂异伍兹烷(CL-20)相当 ${ }^{[43]}$.

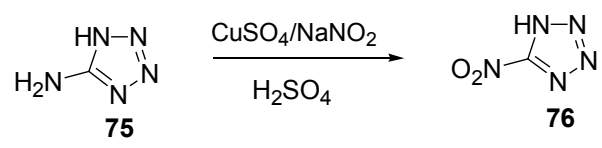

\section{$2.2 \mathrm{NH}_{2}$ 氧化法}

唑类环上的 $\mathrm{NH}_{2}$ 还可经适宜的氧化剂 $\mathrm{F}_{3} \mathrm{CCO}_{3} \mathrm{H}$, $\mathrm{H}_{2} \mathrm{O}_{2}, \mathrm{H}_{2} \mathrm{~S}_{2} \mathrm{O}_{8}$ 等直接氧化成硝基. 如 3-氨基吡唑(77)或 
29 经 $\mathrm{F}_{3} \mathrm{CCO}_{3} \mathrm{H}$ 氧化可分别制得 7 (Eq. 22) ${ }^{[44]}$ 和 3-硝基1,2,4-三唑(78) (Eq. 23) $)^{[45]}$.<smiles>Nc1cc[nH]n1</smiles>

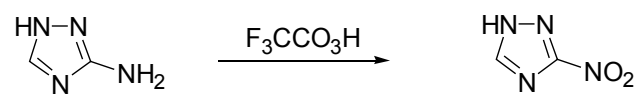

29

78

$\mathrm{H}_{2} \mathrm{O}_{2}$ 和不同试剂混合具有不同的氧化性，与 $\mathrm{H}_{2} \mathrm{SO}_{4}$ 的混合液是很好的强氧化试剂, 如 5-氨基-3,4-二硝基吡 唑(79)经其氧化可制得 10 (3,4,5-TNP) (Eq. 24) ${ }^{[18]}$. 4-氨 基-5-硝基-1,2,3-三唑(80)经 $\mathrm{H}_{2} \mathrm{O}_{2} / \mathrm{H}_{2} \mathrm{SO}_{4}$ 氧化制得 4,5-二 硝 基-1,2,3-三唑(81) (Eq. 25), 其密度为 $1.87 \mathrm{~g} / \mathrm{cm}^{3}$ 、爆 速 $8.88 \mathrm{~km} / \mathrm{s}$ 、爆压 $3.69 \times 10^{7} \mathrm{~Pa}^{[46]}$.<smiles>Nc1[nH]nc([N+](=O)[O-])c1[N+](=O)[O-]</smiles><smiles>Nc1n[nH]nc1[N+](=O)[O-]</smiles><smiles>O=[N+]([O-])c1n[nH]nc1[N+](=O)[O-]</smiles>

$\mathrm{H}_{2} \mathrm{O}_{2}$ 与 $\mathrm{Na}_{2} \mathrm{WO}_{4}$ 混合具有较弱的氧化能力, 表现出 单氧化特性, 与 $\mathrm{F}_{3} \mathrm{CCO}_{3} \mathrm{H}$ 或 $\mathrm{H}_{2} \mathrm{SO}_{4}$ 混合具有较强的氧 化性, 表现出多氧化特性. 如 70 (DAT) 经不同氧化能力 的 $\mathrm{H}_{2} \mathrm{O}_{2}$ 体系氧化可制得 31 (ANTA) 或 71 (DNT) (Scheme 15) ${ }^{[47]}$.

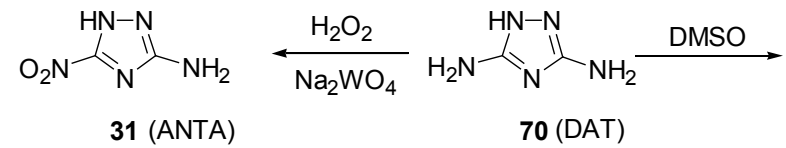

$$
\stackrel{\substack{\mathrm{H}_{2} \mathrm{O}_{2} / \mathrm{F}_{3} \mathrm{CCO}_{3} \mathrm{H} \\ \text { or } \mathrm{H}_{2} \mathrm{O}_{2} / \mathrm{H}_{2} \mathrm{SO}_{4}}}{\longrightarrow} \mathrm{O}_{2} \mathrm{~N} \text { (DNT) }
$$

\section{Scheme 15}

$\mathrm{H}_{2} \mathrm{~S}_{2} \mathrm{O}_{8}$ 也是用于唑类氨基氧化的一种优良试剂, 如 79 或 59 (LLM-116)通过 $\mathrm{H}_{2} \mathrm{~S}_{2} \mathrm{O}_{8}$ 氧化以不同收率制得 10 $(3,4,5-\mathrm{TNP})(\text { Scheme 16 })^{[48]}$. 由于 $\mathbf{5 9}$ 中 4 -位氨基较 79 中 5-位氨基的空间位阻大, 能有效避免分子间副反应的发
生, 氧化反应选择性强, 收率高 ${ }^{[48]}$. 此外, 还有一些氧 化试剂可用于唑类化合物中氨基的氧化，如 3-氯过氧苯 甲酸、 $\mathrm{N}_{2} \mathrm{O}_{5} / \mathrm{H}_{2} \mathrm{SO}_{4}$ 体系等, 此处不再列举.

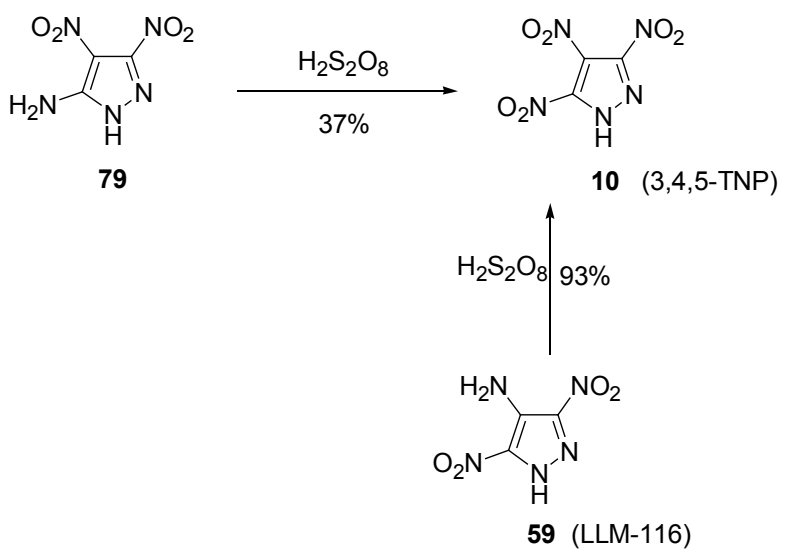

Scheme 16

\section{3 碘代-硝化法}

碘易取代唑环中的氢，同时又易被其他亲电试剂取 代，所以可通过先碘代再硝化的方法制备硝基唑类衍生 物. 其中碘代物的硝化机理为: 亲电基团 $\mathrm{NO}_{2}^{+}$与唑环 形成络合物, 然后选择取代唑环中电子云密度最高的位 置, 最后由于共轭效应的增强, 导致 $\mathrm{C}-\mathrm{I}$ 键或 $\mathrm{N}-\mathrm{I}$ 键 减弱, 最终解离出 $\mathrm{I}^{+}$离子, 形成 $\mathrm{C}-\mathrm{NO}_{2}$ 键或 $\mathrm{N}-\mathrm{NO}_{2}$ 键(Scheme 17). 其发生的芳香亲电取代反应与 Scheme 1 所述大致相同. 碘代一硝化法选择性较高, 易硝化, 反 应条件温和. 通过对单碘代和多碘代唑类化合物的硝化 可制得相应单硝基和多硝基唑类衍生物. 如 7, 8 (3,4-DNP) (Eqs. 26 27), 12 (MTNP) (Scheme 18)的制 备 ${ }^{[49,11]}$.

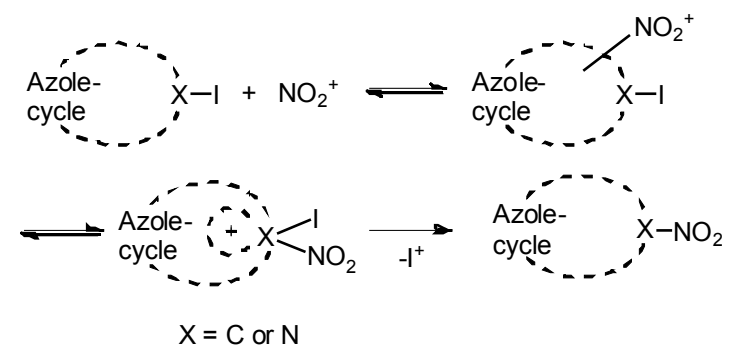

\section{Scheme 17}

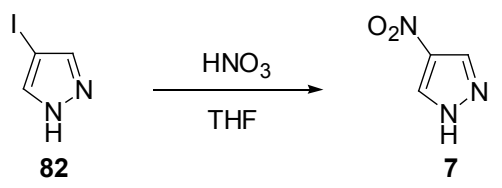

咪唑类, 如 2,4-二碘咪唑 85 经不同的硝化试剂硝化 分别制得单硝基咪唑 $(\mathbf{8 6})$ 和二硝基咪唑 $(\mathbf{8 7})$ (Scheme $19)^{[50]} ; 2,4,5$-三碘咪唑 $(\mathbf{8 8})$ 和 1,2,4,5-四碘咪唑(89)在不 
${ }_{\mathrm{H}}^{\mathrm{I}} \stackrel{\mathrm{HNO}_{3}}{\mathrm{THF}}$

83<smiles>O=[N+]([O-])c1c[nH]nc1[N+](=O)[O-]</smiles>

$8(3,4-D N P)$

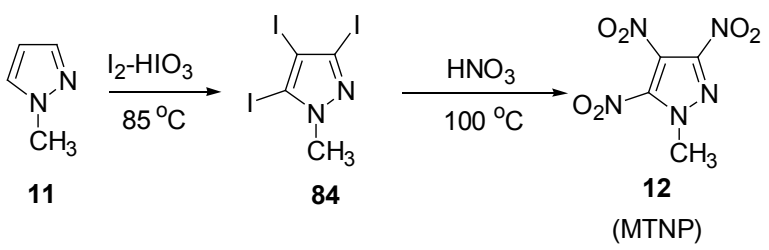

\section{Scheme 18}

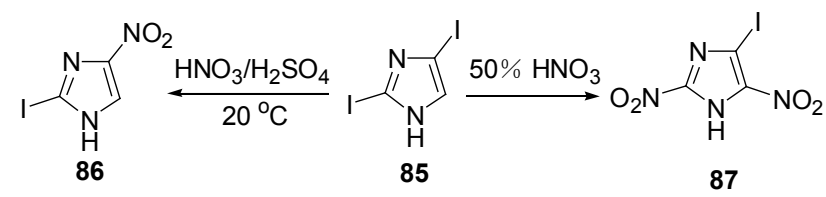

\section{Scheme 19}

同的硝化条件下分别制得单硝基、二硝基和三硝基咪唑 衍生物(Scheme 20) ${ }^{[50,51]}$; 此外, 1-取代-2,4,5-三硝基咪唑 衍生物 36、97 98 的制备, 也可经先碘代再硝化的方法 制得(Scheme 21) ${ }^{[52]}$, 且此方法相对直接硝化法更为容 易，是制备硝基咪唑类衍生物的常用路线之一.<smiles>O=[N+]([O-])c1[nH]c(I)nc1I</smiles>

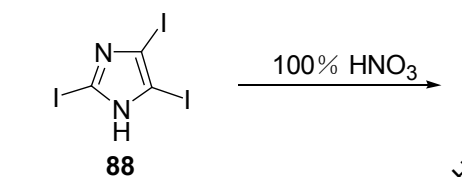<smiles>O=[N+]([O-])c1nc([N+](=O)[O-])c([N+](=O)[O-])[nH]1</smiles>

$18(3,4,5-\mathrm{TNI})$
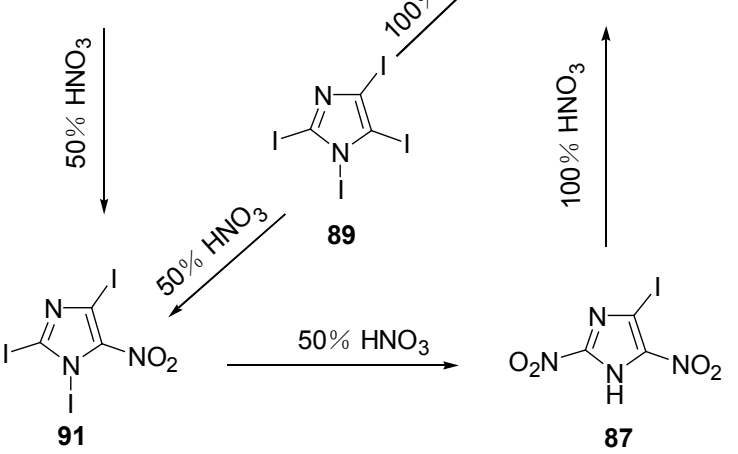

Scheme 20

\section{3 硝基重排法}

由于 $\mathrm{N}-\mathrm{H}$ 键的极性较 $\mathrm{C}-\mathrm{H}$ 键大, $\mathrm{N}-\mathrm{H}$ 键能较小

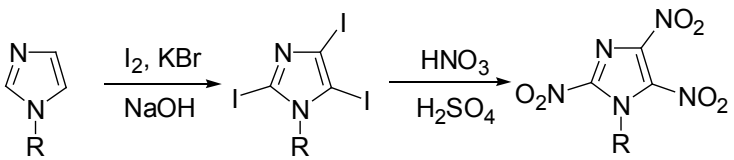

$35 \mathrm{R}=\mathrm{CH}_{3} \quad 94 \mathrm{R}=\mathrm{CH}_{3} \quad 36 \mathrm{R}=\mathrm{CH}_{3}$ $92 \mathrm{R}=\mathrm{CH}_{2} \mathrm{COOC}_{2} \mathrm{H}_{5} 95 \mathrm{R}=\mathrm{CH}_{2} \mathrm{COOC}_{2} \mathrm{H}_{5} 97 \mathrm{R}=\mathrm{CH}_{2} \mathrm{COOC}_{2} \mathrm{H}_{5}$ $93 \mathrm{R}=$ 苦基 $96 \mathrm{R}=$ 苦基 $98 \mathrm{R}=$ 苦基

\section{Scheme 21}

易断裂，经直接硝化法易生成 $\mathrm{N}-\mathrm{NO}_{2}$. 在某些溶剂和 适宜的温度下, $\mathrm{N}-\mathrm{NO}_{2}$ 可发生重排，生成更稳定的 $\mathrm{C}-\mathrm{NO}_{2}$, 可利用此特点实现唑环中碳位引入硝基的目 的. 该法常采用氯苯、苯腈等高沸点溶剂，所需的反应 温度一般较高，反应时间约 2 3 h, 产率一般在 $90 \%$ 左 右.

46 在高沸点溶剂 $\mathrm{PhCN}$ 或 $\mathrm{PhOCH}_{3}$ 中经加热重排制

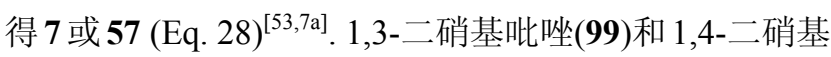
吡唑(47)经硝基重排法可分别制得 9 (3,5-DNP) (Scheme $22)^{[54]}$ 和 8 (3,4-DNP) (Eq. 29) ${ }^{[7 \mathrm{a}]}$.

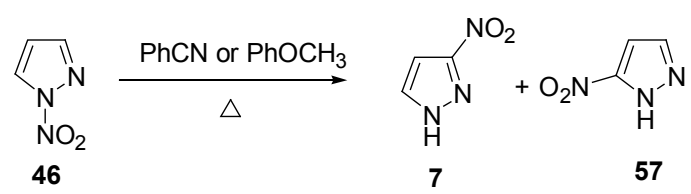

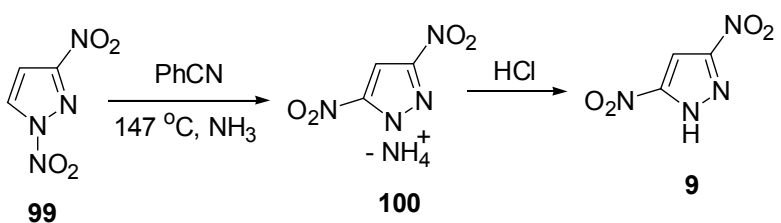

Scheme 22

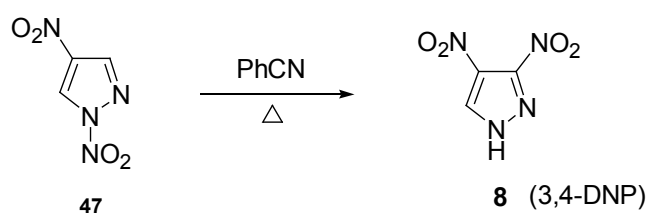

28 (1,4-DNI)中 1-位硝基重排反应受温度影响较大, 在氯苯中于 115 和 $120{ }^{\circ} \mathrm{C}$ 下经重排反应分别制得 $\mathbf{1 7}$

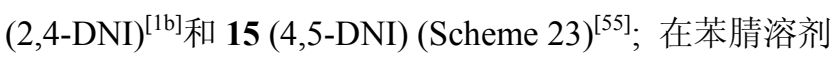
中, 28 经微波照射( $375 \mathrm{~W}, 2 \mathrm{~min}$ )即可重排为 $\mathbf{1 7}^{[56]}$.

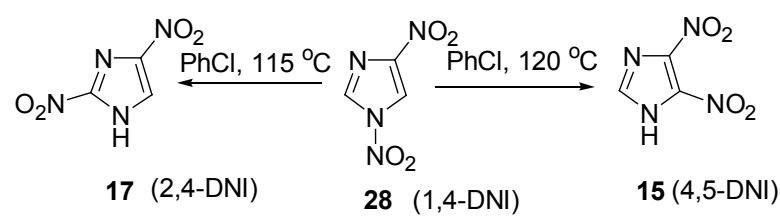

Scheme 23

此外, 一些唑类化合物中的 $\mathrm{N}-\mathrm{NO}_{2}$ 除了向 $\mathrm{C}-\mathrm{NO}_{2}$ 重排外, 还可以重排到其他基团上, 如 $\mathbf{3 0}$ 在苯 
腈中加热，除了生成 31 (ANTA)外，还生成了 3-硝胺基1,2,4-三唑(101) (Eq. 30) ${ }^{[21]}$.

$$
\text { 31 (ANTA) }
$$

\section{4 其他方法}

以上硝化方法是较为常用的制备方法，除此之外还 可采用一些较为特殊的方法, 如环合法、缩环法、其它 基团转化法等合成硝基唑类含能化合物.

\section{1 环合法}

在一定条件下，一些含硝基的开链化合物可发生环 合反应制得硝基唑类化合物. 如硝基吡唑类化合物 7, 6

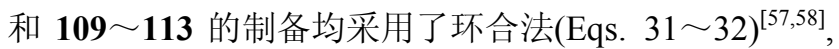
反应条件并不苛刻, 收率不等, 但该法难以实现产物 114 的合成(Eq. 32).

$\mathrm{CH}_{2} \mathrm{~N}_{2}+\mathrm{ClHC}=\mathrm{CHNO}_{2} \stackrel{-\mathrm{HCl}}{\longrightarrow} \overbrace{7}^{\mathrm{NO}_{2}}$<smiles>N/C=C(/C=O)[N+](=O)[O-]</smiles><smiles>COc1ccccc1N</smiles>

$$
\begin{array}{lc}
102 \sim 108 & 6 \mathrm{R}=\mathrm{H} \\
\mathrm{R}=\mathrm{H}, \mathrm{Me}, t-\mathrm{Bu}_{1} \mathrm{CH}_{2} \mathrm{COOEt}, \mathrm{C}_{6} \mathrm{H}_{5}, & 109 \sim 114 \\
4-\mathrm{MeC}_{6} \mathrm{H}_{4}, 4-\mathrm{NO}_{2} \mathrm{C}_{6} \mathrm{H}_{4} & \mathrm{Re}, t-\mathrm{Bu}_{4} \mathrm{CH}_{2} \mathrm{COOEt}, \mathrm{C}_{6} \mathrm{H}_{5}, \\
& 4-\mathrm{MeC}_{6} \mathrm{H}_{4}, 4-\mathrm{NO}_{2} \mathrm{C}_{6} \mathrm{H}_{4}
\end{array}
$$

\begin{tabular}{lccc}
\hline \multicolumn{1}{c}{$\mathrm{R}$} & $T /{ }^{\circ} \mathrm{C}$ & Time/h & Yield/\% \\
\hline $\mathrm{H}$ & r.t. & 3 & 87 \\
$\mathrm{Me}$ & r.t. & 3 & 96 \\
$t-\mathrm{Bu}$ & r.t. & 24 & 29 \\
$t-\mathrm{Bu}$ & 80 & 3 & 59 \\
$\mathrm{CH}_{2} \mathrm{CO}_{2} \mathrm{Et}$ & r.t. & 24 & 90 \\
$\mathrm{C}_{6} \mathrm{H}_{5}$ & 80 & 3 & 35 \\
$4-\mathrm{MeC}_{6} \mathrm{H}_{4}$ & 80 & 3 & 47 \\
$4-\mathrm{NO}_{2} \mathrm{C}_{6} \mathrm{H}_{4}$ & 80 & 3 & 0 \\
\hline
\end{tabular}

以化合物 115 为原料, 在醋酐中经环合法可制备 4硝基-2-苯基-1,2,3-三唑(116) (Eq. 33 ${ }^{[59]}$. 1,1'-二硝基-2芳基乙烯 117～119、1-溴-1-硝基-2-芳基乙烯 120～122 和 $\mathrm{NaN}_{3}$ 于室温下环合制得 4-芳基-5-硝基-1,2,3-三唑 123 125 (Scheme 24), 产率 59\% 77\% ${ }^{[60]}$. 4-硝基-1-苯 基-1,2,3-三唑(127)和 5-硝基-1-苯基-1,2,3-三唑(128)的制 备也采用了该方法(Eq. 34) ${ }^{[61]}$.

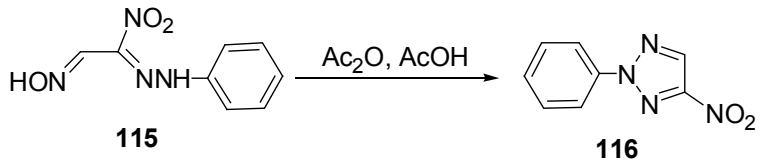

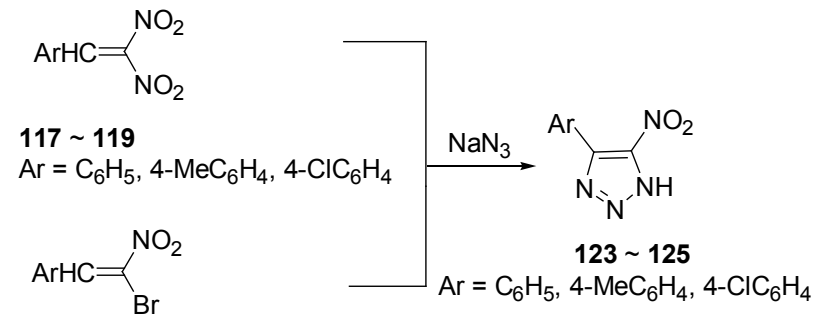

$120 \sim 122$

$\mathrm{Ar}=\mathrm{C}_{6} \mathrm{H}_{5}, 4-\mathrm{MeC}_{6} \mathrm{H}_{4}, 4-\mathrm{ClC}_{6} \mathrm{H}_{4}$

Scheme 24

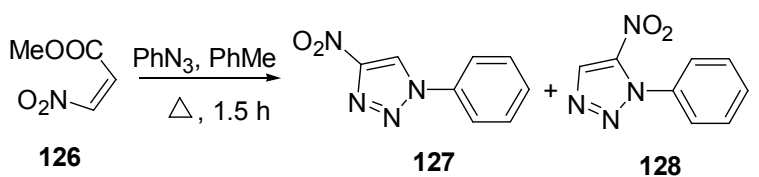

以丙二酸单乙酯(129)为原料, 经 $\mathrm{HNO}_{3} / \mathrm{NO}_{2}$ 硝化制 得二硝基乙酸乙酯(130), 130 和叠氮钠、水、乙醛经环 合反应制得 4-甲基-5-硝基-1,2,3-三唑(131), 再经过氧 化、酯化、酰胺化、Hoffman 降解一系列反应可制备出 80 (Scheme 25) ${ }^{[62]} .80$ 含氮量 54\%、热分解点 $297{ }^{\circ} \mathrm{C}$ 、 $H_{50}=154 \mathrm{~cm}$ ( $2.5 \mathrm{~kg}$ 落锤), 是较为理想的新型针感高能 炸药候选物, 也是合成一些硝基三唑类高能钝感炸药 (如 81) 的重要中间体 ${ }^{[63]}$. 此外通过环合法还可直接制得 5,5'-二硝基-4,4'-联(1,2,3-三唑) (136) (Eq. 35) ${ }^{[64]}$.
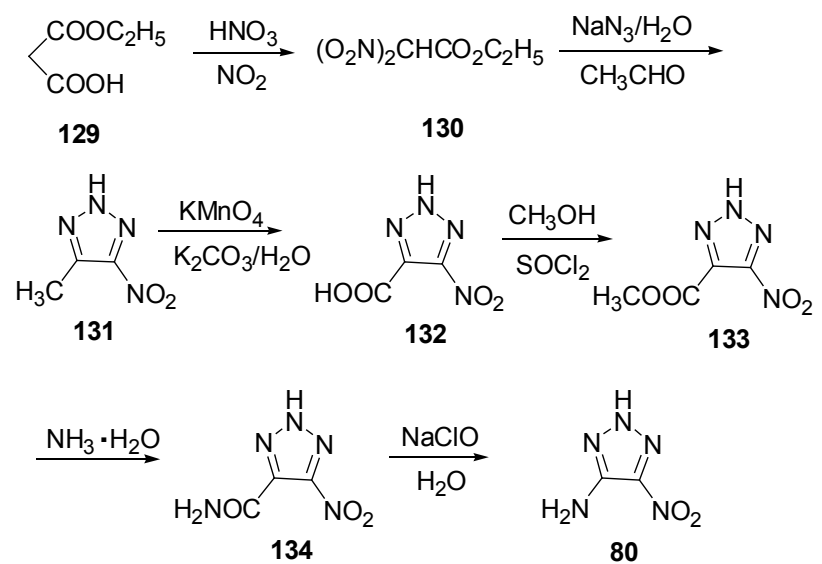

Scheme 25<smiles>O=C([O-])OC(C(C(OC(=O)[O-])[N+](=O)[O-])[N+](=O)[O-])[N+](=O)[O-]</smiles><smiles>O=[N+]([O-])c1n[nH]nc1-c1n[nH]nc1[N+](=O)[O-]</smiles> 


\section{2 缩环法}

一些结构特殊的六元环, 在一定条件下可通过缩环 反应制备出硝基吡唑衍生物. 如 3,5-二硝基吡啶-2-酮 (137)和肼于 $90 \sim 95{ }^{\circ} \mathrm{C}$ 发生缩环反应制得 6 (Eq. 36) ${ }^{[65]}$; 嘧啶衍生物也可与肼发生反应, 制备出相应的五元环硝 基吡唑衍生物, 如 6-氯- $N, N$-二甲基-5-硝基嘧啶-4-胺 (139)与肼在乙醇溶液中加热缩环制得 3,5-二氨基-4-硝 基吡坐(140) (Eq. 37) ${ }^{[66]}$. 4-羊基-5-硝基-2-苯基哒溙-3-酮 (141)和甲胺、水在加热条件下缩环制得 3-硝基-1-苯基 吡唑(142), 而与氢、氨水、铜在 $80{ }^{\circ} \mathrm{C}, 1.01 \mathrm{MPa}$ 则缩环 生成 112 (Scheme 26) ${ }^{[67]}$. 4-甲氧基-5-硝基嘧啶(143)和 4联氨基-5-硝基嘧啶(144)分别和肼通过缩环反应均可制 得 66 (Scheme 27) ${ }^{[68]}$, 其中 144 发生的缩环反应机理如 Scheme 28 所示.<smiles>NNC(=O)NNC[n+]1cc(N=O)c[nH]1</smiles><smiles>[N+]=[N+]([O-])c1ncnc(Cl)c1[N+](=O)[O-]</smiles>

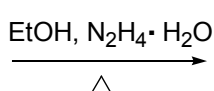<smiles>Nc1n[nH]c(N)c1[N+](=O)[O-]</smiles><smiles>O=Nc1ccn(-c2ccccc2)n1</smiles>

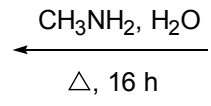<smiles>O=c1c(O)c([N+](=O)[O-])cnn1-c1ccccc1</smiles>

142

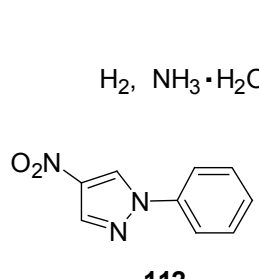

Scheme 26<smiles>O=[N+]([O-])c1cncnc1O</smiles><smiles>N[N+](N)(O)C(=O)O</smiles><smiles>NNc1ncncc1[N+](=O)[O-]</smiles>

$\mathrm{NH}_{2} \mathrm{NH}_{2}$

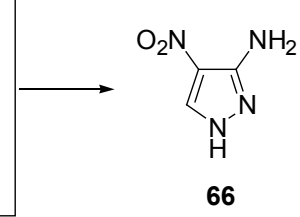

144

Scheme 27

\section{3 其他基团转化法}

对于一些硝基唑类化合物, 分子结构中若存在某些

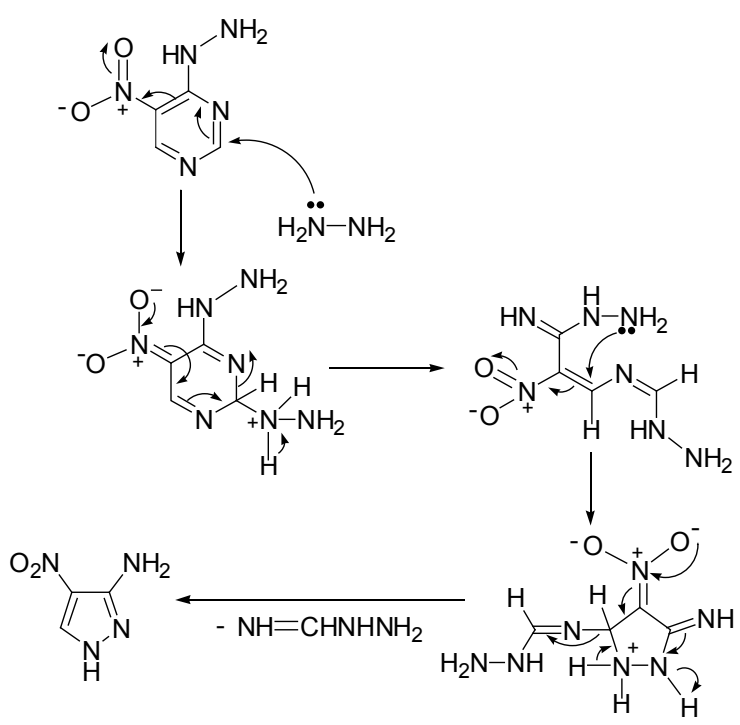

\section{Scheme 28}

特殊基团( $\mathrm{CN}, \mathrm{COOH}$ 等), 也可经适宜的硝化试剂转变 为硝基, 如 3-硝基吡唑-4-腈(145)经 $\mathrm{HNO}_{3} / \mathrm{H}_{2} \mathrm{SO}_{4}$ 硝化, 使氰基转化为硝基制得 8 (3,4-DNP) (Eq. 38) ${ }^{[69]}$. $1 H, 4 H$-3 - 甲基-6-硝基吡唑 [4,3-c]并吡唑(146)经硝化和 氧化制得 148, 其分子中羧基再经 $\mathrm{HNO}_{3}$ 硝化, 可将羒 基直接转化为硝基制得 60 (DNPP) ${ }^{[70]}$. DNPP 含氮量 $42.42 \%$ 、生成焓 $273.0 \mathrm{~kJ} / \mathrm{mol} 、$ 密度 $1.87 \mathrm{~g} / \mathrm{cm}^{3} 、 H_{50}=$ $68 \mathrm{~cm}$ (2.5 kg 落锤), 差示扫描量热法(DSC)分解点 330.8 ${ }^{\circ} \mathrm{C}$, 预测其能量为 HMX 的 $85 \%$. DNPP-铵盐具有较高的 含氮量和 $\Delta H_{\mathrm{f}}$ 值, 燃烧物为碱性物质, 可作为火焰配方 抑制剂和燃速改良剂(Scheme 29) ${ }^{[71]}$.<smiles>N#Cc1c[nH]nc1[N+](=O)[O-]</smiles><smiles></smiles>

145<smiles>Cc1nn[nH]c1CCC(=O)O</smiles><smiles></smiles><smiles>O=[N+]([O-])c1c[nH]nc1[N+](=O)[O-]</smiles><smiles>Cc1n[nH]c2c([N+](=O)[O-])n[nH]c12</smiles>

147<smiles>O=[N+]([O-])c1n[nH]c2c([N+](=O)[O-])n[nH]c12</smiles>

Scheme 29

\section{5 结论与展望}

综上所述，硝基唑类化合物具有较高的密度、氮含 量、化学键能和生成焓, 其中部分化合物还具备了高能 
低(钝)感特性, 是一种研究较为广泛且极具应用价值的 含能材料. 人们相继开发出了诸多硝基唑类化合物的制 备方法, 其中直接硝化法、间接硝化法和硝基重排法较 为常见, 但这些方法仍存在一些局限性, 有必要继续研 究以发现新的合成方法来适应新的需求, 特别是更注重 研发一些低毒、廉价、高效又绿色环保的合成策略, 以 适应可持续发展战略的实施和绿色化学化工的实际应 用. 相信不久的将来, 以硝基唑类化合物为核心的含能 材料将备受关注, 随着理论计算等相关工作的深入研 究, 性能更为优异的各种硝基唑类含能化合物将不断涌 现, 使其在高能钝感炸药、熔铸炸药、推进剂、烟火剂 及其他领域有更加广泛的应用.

\section{References}

[1] (a) Philip, F. P.; Gregory, S. L.; Alexander, R. M.; Robert, D. S. Thermochim. Acta 2002, 384, 187.

(b) Cho, J. R.; Cho, S. G.; Kim, K. J.; Kim, J. K. Insensitive Munition and Engergetic Materials Technology Symposium, Enschede, 2000, pp. 393 400

(c) Tang, Z.; Yang, L.; Qiao, X. J.; Zhang, T. L.; Zhang, J. G.; Liang, Y. H. Acta Chim. Sinica 2012, 70, 471 (in Chinese).

(汤崭, 杨利, 乔小晶, 张同来, 张建国, 梁彦会, 化学学报, 2012, 70, 471.)

(d) Klapotke, T. M.; Gobel, M.; Stierstorfer, J. Int. J. Energ. Mater. Chem. Propul. 2011, 10, 45.

(e) Thottempudi, V.; Forohor, F.; Parrish, D. A.; Shreeve, J. M. Angew. Chem., Int. Ed. 2012, 51, 9881.

(f) Fischer, N.; Klapötke, T. M.; Reymann, M.; Stierstorfer, J. Eur. J. Inorg. Chem. 2013, 2013, 2167.

(g) Ek, S.; Rehn, S.; Wahlström, L. Y.; Östmark, H. J. Heterocycl. Chem. 2013, 50, 261.

[2] (a) Li, Y. J.; Cao, D. L.; Li, Y. X.; Du, Y.; Wang, J. L. Chin. J. Explos. Propellants 2013, 36, 28 (in Chinese).

(李雅津, 曹端林, 李永祥, 杜耀, 王建龙, 火炸药学报, 2013, 36,28 .)

(b) Chai, C. P.; Gan, Z. Y.; Li, N.; Luo, Y. J. Chin. J. Explos. Propellants 2011, 34, 36 (in Chinese).

(柴春鹏, 甘志勇, 李娜, 罗运军, 火炸药学报, 2011, 34, 36.)

(c) Badgujar, D. M.; Talawar, M. B.; Asthana, S. N.; Mahulikar, P. P. J. Hazard. Mater. 2008, 151, 289.

[3] (a) Zhang, Y. Q.; Parrish, D. A.; Shreeve, J. M. J. Mater. Chem. A 2013, 1,585 .

(b) Chen, B. R.; Li, J. R.; Ou, Y. X. Chin. J. Explos. Propellants 1992, 3, 26 (in Chinese).

(陈博仁，李加荣，欧育湘，火炸药学报, 1992, 3, 26.)

(c) Kurpet, M.; Jędrysiak, R.; Suwiński, J. Chem. Heterocycl. Compd. 2013, 48, 1737.

(d) Larina, L.; Lopyrev, V. Nitroazoles: Synthesis, Structure and Applications, Springer, New York, 2009, pp. 5 64.

(e) Sasidharan, N.; Arolickal, G. R. Propellants, Explos., Pyrotech. 2012, 37, 267.

(f) Vereschagin, L. I.; Pokatilov, F. A.; Kizhnyaev, V. N. Chem. Heterocycl. Compd. 2008, 44, 1.

(g) Gümüşa, S. J. Energ. Mater. 2012, 30, 156.

[4] Lee, K. Y.; Chapman, L. B.; Cobura M. D. J. Energ. Mater. 1987, $5,27$.

[5] (a) Nair, U. R.; Asthana, S. N.; Rao, A. S.; Gandhe, B. R. Def. Sci.
J. 2010, 60, 137

(b) Xue, H.; Gao, H. X.; Twamley, B.; Shreeve, J. M. Chem. Mater. 2007, 19, 1731.

[6] Luo, Y. F.; Ge, Z. X.; Wang, B. Z.; Zhang, H. H.; Liu, Q. Chin. J. Energ. Mater. 2007, 15, 205 (in Chinese).

(罗义芬, 葛忠学, 王伯周, 张海吴, 刘惩, 含能材料, 2007, 15, 205.)

[7] (a) Janssen, J. W. A. M.; Koeners, H. J.; Kruse, C. G.; Habraken, C. L. J. Org. Chem. 1973, 38, 1777.

(b) Wang, X. Y.; Liu, S. Q.; Zhang, C. Y.; Song, G.; Bai, F. Y.; Xing, Y. H.; Shi, Z. Polyhedron 2012, 47, 151.

[8] (a) Price, D.; Morris, J. 2010 Insentive Munitions and Energetion Materials Technology Symposium, Holston, 2009.

(b) Reddy, D.; Surapaneni, C. R.; Gelber, N.; Duddu, R. G.; Zhang, M. X.; Dave, P. R. US 7304164, 2007 [Chem. Abstr. 2007, 148, 36047].

[9] Dalinger, I. L.; Vatsadze, I. A.; Shkineva, T. K.; Popova, G. P.; Shevelev, S. A. Mendeleev Commun. 2010, 20, 253.

[10] Ravi, P.; Gore, G. M.; Venkatesan, V.; Tewari, S. P.; Sikder, A. K. J. Hazard. Mater. 2010, 183, 859.

[11] Ravi, P.; Chaganti, K. R.; Ananta, S.; Girish, M. G.; Arun, K. S.; Surya, P. T. Propellants, Explos., Pyrotech. 2012, 37, 167.

[12] Li, Y. X.; Wang, J. L.; Cao, D. L.; Liu, L. L.; Song, L. Chin. J. Explos. Propellants 2011, 34, 35 (in Chinese).

(李永祥, 王建龙, 曹端林, 刘丽丽, 宋否, 火炸药学报, 2011, 34,35 .)

[13] Cho, J. R.; Kim, K. J.; Cho, S. G.; Kim, J. K. J. Heterocycl. Chem. 2002, 39, 141

[14] (a) Smiglak, M.; Hines, C. C.; Wilsion, T. B.; Rogers, R. D.; Singh, S.; Vincek, A. S.; Kirichenko, K.; Katritzky, A. R. Chem.-Eur. J. 2010, 16, 1572.

(b) Katritzky, A. R.; Singh, S.; Kirichenko, K.; Smiglak, M.; Holbrey, J. D.; Reichert, W. M.; Spear, S. K.; Rogers, R. D. Chem.Eur. J. 2006, 12, 4630.

[15] Gao, H. X.; Ye, C. F.; Gupta, O. D.; Xiao, J. C.; Hiskey, M. A.; Twamley, B.; Shreeve, J. M. Chem. -Eur. J. 2007, 13, 3853.

[16] (a) Neuman, P. N. J. Heterocycl. Chem. 1971, 8, 51.

(b) Eagles, T. E.; Khan, M. A.; Lynch, B. M. Org. Prep. Proced. 1970, 2, 117

[17] Katritzky, A. R.; Scriven, E. F. V.; Majumder, S.; Akhmedova, R. G.; Akhmedov, N. G.; Vakulenko, A. V. ARKIVOC 2005, (iii), 179.

[18] Dalinger, I. L.; Popova, G. P.; Vatsadze, I. A.; Shkineva, T. K.; Shevelev, S. A. Russ. Chem. Bull. 2009, 58, 2185.

[19] (a) Damavarpu, R.; Jayasuriya, K.; Vladimiroff, T.; Randolph, S. I. US 5387297, 1995 [Chem. Abstr. 1995, 122, 239709].

(b) Luo, M. K.; Singh, V.; Taylor, E. A.; Schramm, V. L. J. Am. Chem. Soc. 2007, 129, 8008.

[20] Lancini, G. C.; Maggi, N.; Sensi, P. Farmaco (Pavia) Ed. Sci. 1963, $18,390$.

[21] Pevzner, M. S.; Kulibabina, T. N.; Povarova, N. A.; Kilina, L. V. Khim. Geterotsiki. Soedin. 1979, 8, 1132.

[22] (a) Sikder, A. K.; Geetha, M.; Sarwade, D. B.; Agrawal, J. P. J. Hazard. Mater. 2001, 82, 1.

(b) Wartenberg, C.; Charrue, P.; Laval, F. Propellants, Explos., Pyrotech. 1995, 20, 23.

(c) Jia, S. Y.; Wang, B. Z.; Hao, C. G.; Hai, H.; Zhou, Q.; Wang, X. J. Chin. J. Explos. Propellants 2012, 35, 23 (in Chinese).

(贾思媛, 王伯周, 郝成刚, 海吴, 周群, 王锡杰, 火炸药学报, 2012, 35, 23.)

[23] Lee, K. Y. Los Alamos National Laboratory, Report LA-10346-MS, New Mexico, USA, 1985.

[24] Herve, G. US 2009/0186931, 2009 [Chem. Abstr. 2009, 151, 
201533].

[25] Ravi, P.; Tewari, S. P. Propellants, Explos., Pyrotech. 2012, 37, 544.

[26] (a) Glass, R. S.; Blount, J. F.; Butler, D.; Perrotta, A.; Oliveto, E. P. Can. J. Chem. 1972, 50, 3472.

(b) Pevzner, M. S.; Kulibabina, T. N.; Ioffe, S. L.; Maslina, I. A.; Gidaspov, B. V.; Tartakovskii, V. A. Chem. Heterocycl. Compd. 1979, 15, 451.

[27] Brusnikina, V. M. Khim. Geterotsiki. Soedin. 1963, 167.

[28] Lv, C. X. Chin. J. Explos. Propellants 2011, 34, 1 (in Chinese). (吕春绪, 火炸药学报, 2011, 34, 1.)

[29] Suzuki, H.; Nonoyama, N. J. Chem. Res., Synop. 1996, 244.

[30] (a) Cheng, J. M. S. Thesis, Nanjing University of Science and Technology, Nanjing, 2007 (in Chinese). (成健, 硕士论文，南京理工大学，南京，2007.)

(b) Claridge, R. P.; Lancaster, N. L.; Millar, R. W.; Moodie, R. B.; Sandall, J. P. B. J. Chem. Soc., Perkin Trans. 2 2001, 197.

[31] Lebedv, V. P.; Matyushim, Y. N.; Inolemtcev, Y. D. Int ICT Conference on Energetic Materials, Russia, 1998, p. 180.

[32] Schmidt, R. D.; Lee, G. S.; Pagoria, P. F.; Mitchell, A. R.; Gilardi, R. J. Heterocycl. Compd. 2001, 38, 1227.

[33] Luo, Y. F.; Wang, B. Z.; Chen, X. F.; Li, Y. N.; Li, W. J. Chin. J. Energ. Mater. 2012, 20, 814 (in Chinese).

(罗义芬, 王伯周, 陈晓芳, 李亚南, 李文杰, 含能材料, 2012, 20,814.)

[34] Chavez, D. E.; Parrish, D. A. Propellants Explos., Pyrotech. 2012, $37,536$.

[35] Liu, M. H.; Wu, M. Y. Open J. Phys. Chem. 2012, 2, 107.

[36] Ravi, P.; Tewari, S. P. Catal. Commun. 2012, 19, 37.

[37] Latypov, N. V.; Silevivh, V. A.; Lvanov, P. A.; Pevzner, M. S. Chem. Heterocycl. Compd. 1976, 12, 1355.

[38] Hiskey, M.; Chavez, D. E.; Naud, D. L.; Son, S. F.; Berghout, H. L.; Bolme, C. A. In Proceedings of the 27th International Pyrotechnic Seminar, Grand Junction, 2000, pp. 1 14.

[39] Xiong, C. L.; Jia, S. Y.; Wang, X. J.; Wang, B. Z.; Zhang, Y. G. Fine Chem. Intermed. 2008, 38, 64 (in Chinese).

(熊存良, 贾思媛, 王锡杰, 王伯周, 张叶高, 精细化工中间体, 2008, 38, 64.)

[40] Lei, F. B.; Zhu, J. P.; Cao, D. L. Introd. J. China Ordnance 2011, 32,8 (in Chinese). (雷锋斌, 朱佳平, 曹端林, 兵工学报, 2011, 32, 8.)

[41] Bagal, L. I.; Pevzner, M. S. Chem. Heterocycl. Compd. 1970, 6, 517.

[42] Li, Y.; Liu, W.; Pang, S. Molecules 2012, 17, 5040.

[43] Li, Z. M.; Zhang, J. G.; Zhang, T. L.; Shu, Y. J. Prog. Chem. 2010, 22, 639 (in Chinese).

(李志敏, 张建国, 张同来, 舒远杰, 化学进展, 2010, 22, 639.)

[44] Lebedav, O. V. Indian J. Chem. 1976, 7, 455.

[45] Namesmikov, V. I.; Kofinan, T. P.; Pevzner, M. S. SU 1027159, 1983 [Chem. Abstr. 1983, 99, 194973].

[46] (a) Baryshnikov, A. T.; Erashko, V. I.; Zubanova, N. I.; Ugrak, B. I.; Shevelev, S. A.; Fainzil'berg, A. A.; Laikhter, A. L.; Mel'nikova, L. G.; Semenov, V. V. Bull. Russ. Acad. Sci. 1992, 41, 751.

(b) Zhang, Y. Q.; Parrishb, D. A.; Shreeve, J. M. J. Mater. Chem. A 2013, 1,585 .

[47] Taylor, E. C.; Tseng, C. P.; Rampal, J. B. J. Org. Chem. 1982, 47, 552.

[48] Hervé, G.; Roussel, C.; Graindorge, H. Angew. Chem. 2010, 122, 3245.

[49] Ravi, P.; Tewari, S. P.; Gore, G. M.; Sikder, A. K. Synth. Commun. 2012, 42, 3463.

[50] Novikov, S. S.; Khmel'nitskii, L. I.; Lebedev, O. V.; Epishina, L.
V.; Sevost'yanova, V. V. Chem. Heterocycl. Compd. 1970, 6, 614.

[51] Katritzky, A. R.; Cundy, D. J.; Chen, J. J. Energ. Mater. 1993, 11, 343.

[52] Jadhav, H. S.; Talawar, M. B.; Sivabalan, R.; Dhavalea, D. D.; Asthanab, S. N.; Krishnamurthya, V. N. J. Hazard. Mater. 2007, 143, 192.

[53] Janssen, J. W. A. M.; Habraken, C. L. J. Org. Chem. 1971, 36, 3081.

[54] Wang, Y. L.; Zhang, Z. Z.; Wang, B. Z.; Zheng, X. D.; Zhou, Y. S. Chin. J. Energ. Mater. 2007, 15, 574 (in Chinese). (汪营磊, 张志忠，王伯周，郑晓东，周彦水，含能材料，2007, 15, 574.)

[55] Fassakhov, R. K.; Sirzyanov, G. R.; Nallmov, V. V. SU 101164, 1979 [Chem. Abstr. 1957, 51, 68625].

[56] Bhaumik, K.; Akamanchi, K. G. J. Heterocycl. Chem. 2004, 41, 51.

[57] Verbruggen, R.; Viehe, H. G. Chimia 1975, 29, 350.

[58] (a) Nishiwaki, N.; Ogihara, T.; Takami, T.; Tamura, M.; Ariga, M. J. Org. Chem. 2004, 69, 8382.

(b) Nishiwaki, N.; Tohda, Y.; Ariga, M. Bull. Chem. Soc. Jpn. 1996, 69, 1997.

[59] Krivopalov, V. P.; Shkurko, O. P. Russ. Chem. Rev. 2005, 74, 339.

[60] Sheremet, E. A.; Tomanov, R. I.; Trukhin, E. V.; Berestovitskaya, V. M. Russ. J. Org. Chem. 2004, 40, 594.

[61] (a) Makarova, N. G.; Anisimova, N. A.; Berkova, G. A.; Deiko, L. I.; Berestovitskaya, V. M. Russ. J. Org. Chem. 2005, 41, 941.

(b) Berestovitskaya, V. M.; Anisimova, N. A.; Kataeva, O. N.; Makarova, N. G.; Berkova, G. A. Russ. J. Gen. Chem. 2007, 77, 1567.

[62] Pagoria, P. F.; Lee, G. S.; Mitchell, A. R.; Schmidt, R. D. Insensitive Munitions and Energetic Materials Technology Symposium, Bordeaux, France, 2001, pp. 655 661 .

[63] Huo, H.; Wang, B. Z.; Zhou, C.; Xiong, C. L. Chin. J. Energ. Mater. 2008, 16, 49 (in Chinese). (霍欢, 王伯周, 周诚, 熊存良, 含能材料, 2008, 16, 49.)

[64] Baryshnikov, A. T.; Erashko, V. I.; Zubanova, N. I.; Ugrak, B. I.; Shevelev, S. A.; Fainzilberg, A. A.; Semenov, V. V. Bull. Russ. Acad. Sci. 1992, 41, 1657.

[65] Yutilov, Y. M.; Smolyar, N. N. Chem. Heterocycl. Compd. 1986 22,926 .

[66] Guillard, J.; Goujon, F.; Badol, P.; Poullain, D. Tetrahedron Lett. 2003, 44, 5943.

[67] Predvoditeleva, G. S.; Kartseva, T. V.; Shchukina, M. N. Pharm. Chem. J. 1974, 8, 525.

[68] (a) Biffin, M. E. C.; Brown, D. J.; Porter, Q. N. Tetrahedron Lett. 1967, 8, 2029

(b) Biffin, M. E. C.; Brown, D. J.; Porter, Q. N. J. Chem. Soc. (C) $1968,8,2159$.

[69] Vinogradov, V. M.; Cherkasova, T. I.; Dalinger, I. L.; Shevelev, S. A. Russ. Chem. Bull. 1993, 42, 1552.

[70] (a) Fukata. G.; Kawazoe, Y.; Taguchi, T. Yakugaku Zasshi 1974, 94, 23.

(b) Vinogradov, V. M.; Dalinger, I. L.; Shevelev, S. A. Mendeleev Commun. 1993, 3, 111

[71] (a) Li, Y. N.; Tang, T.; Lian, P.; Luo, Y. F.; Yang, W.; Wang, Y. B.; Li, H.; Zhang, Z. Z.; Wang, B. Z. Chin. J. Org. Chem. 2012, 32, 580 (in Chinese).

(李亚南, 唐婷, 廉鹏, 罗义芬, 杨威, 王友兵, 李辉, 张志忠, 王伯周, 有机化学, 2012, 32, 580.)

(b) Stern, A. G.; Moran, J. S.; Jouet, R. J.; Sitzman, M. E.; Pagoria, P. F.; Lee, G. S. US 6706889, 2004 [Chem. Abstr. 2004, 140, 255814].

(Zhao, X.) 